Published in final edited form as:

Curr Opin Nephrol Hypertens. 2016 November ; 25(6): 570-576. doi:10.1097/MNH.0000000000000262.

\title{
Current status of the microbiome in renal transplantation
}

\author{
Sarwat Ahmad, MD and Jonathan Bromberg, MD, PhD* \\ Department of Surgery, University of Maryland Medical Center, Baltimore, MD
}

\begin{abstract}
Purpose of Review: An imbalance between pathogenic and protective microbiota characterizes dysbiosis. Presence of dysbiosis may affect immunity, tolerance, or disease depending on a variety of conditions. In the transplant patient population, the need for immunosuppression and widespread use of prophylactic and therapeutic antimicrobial agents creates new post-transplant microbiota communities that remain to be fully defined.
\end{abstract}

Recent Findings: Studies in mice have demonstrated significant bidirectional interactions between microbiota derived products and host immune cells. The stimulation of Treg and Th17 cells by specific products leads to maintenance of immune homeostasis versus activation of inflammation, respectively. Dysbiosis may lead to development of antigen cross-reactivity, which may affect alloreactivity. Certain immunologic sequelae of microbiota are pronounced in chronic kidney disease, due to uremia and renal metabolism of microbiota metabolites. Dietary modifications, probiotics, and fecal microbiota transplant have been investigated for alteration of microbiota in humans.

Summary: Researchers have begun to identify dysbioses associated with clinical conditions, including chronic kidney disease, post-transplant infection, and rejection. This information will allow clinicians not only to select at-risk patients for early intervention, but also to develop therapies that restore the microbiota to a state of homeostasis or tolerance..

\section{Keywords}

Microbiota; microbiome; chronic kidney disease; transplantation

\section{Introduction}

The vast community of microorganisms that exists on our epithelial surfaces is collectively termed the microbiota. The microbiome refers to the microbiota plus the surrounding environment. The microbiota is able to induce disease [1-3] and also able to regulate immunity or tolerance [4-7]. An imbalance between pathogenic and protective microbes characterizes dysbiosis, a perturbation in the normal composition of commensal microbiota [8]. Examples of conditions resulting from and/or contributing toward dysbiosis include Clostridium difficile [1], Crohn's disease [2, 9], and non-alcoholic steatohepatitis (NASH)

\footnotetext{
*Correspondence: Jonathan S. Bromberg, Department of Surgery, University of Maryland School of Medicine, 29 S. Greene Street, Suite 200, Baltimore, MD 21201, jbromberg@ smail.umaryland.edu, Phone: 410328 0008, Fax: 4103286343.

Conflicts of Interest: none
} 
[3]. In NASH, enterohepatic portal flow enables direct transfer of microbe and immune system derived inflammatory mediators from intestine to liver, leading to the activation of several molecular pathways. Henao-Mejia et al. identified that inflammasome-deficient mice had expanded Bacteroidetes and experienced an influx of lipopolysaccharide into the portal circulation, leading to increased hepatic tumor-necrosis factor and exacerbation of the NASH phenotype [10]. Thus, gut dysbiosis may transfer immune cells and signals to distant sites, rendering extraintestinal tissues such as organ allografts susceptible to changes in the intestinal microbiota.

In transplant patients, immunosuppression, metabolic abnormalities, and antibiotics mediate profound alterations of microbiota. This review examines this dynamic relationship at immunologic and clinical levels and presents the evidence to date describing the impact of the microbiota in renal transplantation.

\section{Studying the Microbiome}

The thousands of species and the millions of genes expressed by the microbiota (i.e. metagenome) are a reflection of the functional diversity required to maintain microbiota homeostasis. This vast array of gene expression has been studied by many groups to identify microbiome conditions that lead to health versus disease. Genetic tools to characterize microbiota include targeted sequencing of the 16S ribosomal RNA genes and shotgun sequencing of transcribed genes [11] [Table 1]. The huge amount of information obtained can be processed using principal coordinate analyses and classical multidimensional scaling [12]. Briefly, the sequenced 16s rRNA dataset is processed by phylogenetic classification to measure relative abundance of the bacterial taxa in each sample. Using extended local similarity analysis (eLSA) [13] and linear discriminant analysis effect size (LefSe) [14], one can measure differences between samples in terms of biologic diversity and biomarker expression. The Human Microbiome Project [15] and European MetaHit Consortium [16] have identified $99 \%$ of the genera present in normal human microgenomes in Western populations for investigators to use as a template for classifying individual samples or large populations.

\section{Mechanisms of Microbiota Stimulation of the Enteric Immune System}

The microbiota interacts with the intestinal epithelial cells (IEC) and resident immune cells to activate both innate and adaptive mechanisms [Figure 1]. Microbial products are sensed by receptors of the IEC, such as toll-like receptor (TLR) and nucleotide-binding oligomerization domain (NOD). For example, peptidoglycan from gram negative bacteria binds NOD1 and elicits production of CCL20 and $\beta$-defensin- 3 to recruit B cells to the lamina propria (LP) and induce expression of IgA [17]. Development of intestinal lymphoid follicles depends on the presence of both microbial products and lymphoid tissue inducer cells (LTi), a subset of innate lymphoid cells (ILC), in the LP [18]. At homeostasis, bacterial products stimulate production of mucus, bactericidal molecules, and biofilms by IEC to exclude pathogenic bacteria and promote colonization by commensal bacteria [19, 20]. Virulence factors may penetrate the mucus and activate innate effectors in the LP such as natural killer T cells [21] and ILC. In response to IEC-derived pro-inflammatory cytokines, 
ILC release IL-22 to help maintain the integrity of the epithelium and produce antimicrobial peptides [22]. In contrast, commensal bacteria provide tonic stimulation of the apical receptors to dampen the inflammatory response by various adaptive mechanisms. For example, Bacteroides and Lactobacillus species inhibit activation of the classical NF- $\mathrm{kB}$ pathway and its downstream pro-inflammatory genes [23].

Products from microbiota engage the adaptive immune system by either directly interacting with DC or inducing IEC cytokine expression that influences recruitment and differentiation of neutrophils, macrophages, or DC [24]. At homeostasis, cytokines such as IL-33, IL-25, TGF $\beta$, and TSLP (thymic stromal lymphopoietin) activate macrophages and DC. In the presence of TGF $\beta$ and retinoic acid, DC promote induced Treg (iTreg). Microbial antigens from B.fragilis and Clostridia species promote Treg development [25, 26]. Antigen-specific iTreg then migrate to lymph nodes, express anti-inflammatory cytokines such as IL-10 and TGF $\beta$, and mediate direct killing or inactivation of effector cells. In this way, iTreg suppress the immune response to commensal organisms and self-antigen at a local and systemic level.

During dysbiosis or mucosal injury, pathogenic microbial products stimulate secretion of pro-inflammatory cytokines (e.g., IL-6, IL-1, IL-23, and IL-12), which induce differentiation of CD4+ Th1 and Th17 cells in the small intestine $[25,26]$. Th17 differentiation is induced in the gut by Candidatus arthromitus or segmented filamentous bacteria (SFB) [27]. Th17 cells bolster the intestinal barrier via IL-22 and prevent infection from pathogenic bacteria via IL-17 and IFN $\Upsilon$ expression, and can thus potentially restore homeostasis in a dysbiotic environment [27].

The balance between anti-inflammatory and pro-inflammatory cells can lead to either suppression or activation of disease. The ability to alternate between pathways depends on the microbes and cytokines present. For example, retinoic acid (a cofactor for both iTreg and Th17 differentiation) is suppressed in the presence of noncommensal organisms and proinflammatory cytokines, thus allowing naïve $\mathrm{T}$ cells to be programmed into inflammatory effectors [28]. Specific antibiotic agents have been shown to block retinoic acid suppression [4]. This demonstrates how changes in microbiota may impact changes in molecular signaling that influence one $\mathrm{T}$ cell pathway over another.

Microbiota regulation of $\mathrm{T}$ cells has been associated with antigen-independent systemic inflammation. For example, intestinal SFB promote the development of rheumatoid arthritis via regulation of Th17 differentiation. This was shown in germ-free mice, where introduction of SFB alone could trigger arthritis via IL-17 expressed by Th17 cells. [4]. These data showing how a single microbe can promote a $\mathrm{T}$ cell subset to drive autoimmune disease demonstrate how gut microbes influence systemic inflammation.

Microbiota-derived systemic inflammation may affect alloimmunity via molecular mimicry driven by antigen cross-reactivity, such as homologous sequences between Kell antigen on red blood cells and B.fragilis [29]. Pantenburg et al. showed that cross reactive, primed alloreactive $\mathrm{T}$ cells led to accelerated skin allograft rejection in mice infected with Leishmania major [30]. This suggests that interactions with microbiota may alter the 
immune responses to alloantigen due to the presence of cross-reactive, environmentally derived antigen.

\section{Bidirectional relationship between microbiota and the immune system after allotransplantation}

While the microbiota clearly influences immune pathways, dysregulated immune responses also impact the microbiome in a bidirectional manner. For example, absence of Treg in mice was associated with pronounced Th2 type inflammation in the gut and airways, as well as altered gut microbiota, suggesting that Treg maintain eubiosis in the gut by regulating Th2 inflammation [31]. Independent of cause, inflammation in the gut leads to changes in microbiota composition [32], leading to dysbiosis. Dysbiosis in turn disrupts the immune thresholds set by "normal" commensals, resulting in chronic, recurrent infection or inflammation and inability to clear "abnormal" microbiota [24].

Host factors in transplant patients that influence this bidirectional relationship include antibiotics, immunosuppression, and chronic kidney disease (CKD). Germ-free or antibiotictreated mice are deficient in Th17 cells [27] and have impaired Treg function [33]. These deficiencies are associated with dysregulation of immunity, inflammation, and response to infection, leading to an increased burden of pathogens and the potential to develop crossreactive alloantibodies. Thus, dysbiosis associated with chronic antibiotic use may have immune consequences in allograft recipients beyond simply the emergence of antibioticresistant organisms.

CKD is associated with several immunologic sequelae that can affect the microbiota [Table 2] [34]; conversely, the microbiota have been linked to clinical and immunologic changes in animal models of CKD [Table 3] [34-44]. The buildup of uremic toxins, fluid overload, and subsequent bowel wall edema lead to increased bacterial translocation across the gut epithelial border, causing chronic systemic inflammation [45, 46]. The microbial metabolite TMAO (trimethylamine-N-oxide) has recently been linked to increased mortality in CKD, as well as progressive renal fibrosis, platelet hyperreactivity, thrombosis, lipid metabolism, and inflammation [35-38]. These data illustrate that renal failure predisposes a host to the influx of microbial metabolites, which may have profound clinical impact on these patients.

Immunosuppression is also linked to to dysbiosis. T cell depleting antibodies (antithymocyte globulin), non-depleting antibodies (basiliximab), and glucocorticoids may produce changes in microbiota [39]. These changes may affect one's ability to restore immunity after lymphodepletion, as compensatory homeostatic $\mathrm{T}$ cell proliferation is partially driven by commensal bacterial antigens [47]. While conventionally raised $\mathrm{T}$ celldeficient mice show rapid $\mathrm{T}$ cell proliferation after adoptive transfer of naïve $\mathrm{T}$ cells, germfree T cell-deficient mice undergo only a slow homeostatic proliferation [48], supporting the role of microbiota in influencing immunity in the chronically immunodeficient patient. 


\section{Clinical manifestations of microbiome disruption}

Differences in microbiota after transplantation can be associated with clinically significant events. Decreased Firmicutes in small bowel recipients has been associated with acute rejection [49]. In lung transplant patients, restoration of microbiota diversity decreased the risk of bronchiolitis obliterans syndrome [51]. Bronchial samples containing greater than $10 \%$ Pseudomonas aeruginosa were associated with symptomatic infection, while those containing greater than $10 \%$ P.fluorescens were not. No sample had greater than $10 \%$ of both species [52]. Such changes, however, are difficult to interpret in the presence of concomitant antimicrobial administration.

Fricke et al. demonstrated that major shifts in microbiota composition (measured in blood, oral, urinary, and rectal samples) were identifiable at one month after renal transplant [53]. In another study of 26 renal transplant recipients, Lee at al. identified increased Proteobacteria species in rectal microbiota at 90 days [54]. Patients with post-transplant diarrhea had reduced microbiota diversity, with reduced Bacteroides, Ruminococcus, Coprococcus, and Dorea. Patients with abundant Enterococcus in rectal stool samples were more likely to have an Enterococcus urinary tract infection [54].

Changes in microbiota composition in immunosuppressed patients may also contribute to emergence of opportunistic infection. In four patients with post-renal transplant infection, there was significantly decreased Anaerotruncus (phylum: Firmicutes) in pre-transplant rectal microbiota, compared to 14 healthy control samples [53]. Absence of Bacteroides and Ruminococcus was associated with development of post-renal transplant diarrhea [54]. As mentioned above, reduced diversity of airway microbiota was associated with acute infection in lung recipients [52].

Changes in microbiota may alter metabolism of antirejection medications [55]. In a study of 19 kidney recipients who submitted rectal samples, those who required more than a 50\% increase in tacrolimus dosing during the first month of transplantation were more likely to host an abundance of fecal Faecalibacterium prausnitzii in the first week post-transplant [55]. Oral bioavailability of tacrolimus depends partially on intestinal CYP3A4 and P glycoprotein function and is altered in renal transplant recipients with diarrhea [56]. While no causal relationship has been demonstrated, it is tempting to speculate that since gut mucosal health is associated with tacrolimus metabolism, perhaps gut microbiota contribute to drug metabolism via maintenance or disruption of mucosal integrity.

\section{Therapeutic or diagnostic options under investigation}

Characterizing microbial shifts and biomarkers associated with rejection or infection may identify patients who would benefit from earlier intervention or surveillance. This may also assist development of therapeutics that modulate the immune system to promote allograft acceptance or tolerance. Recent studies have explored the use of fecal microbiota transplant (FMT), probiotics, and dietary modifications.

Diet impacts the availability of metabolites required for intestinal immune cells. For example, retinoic acid is required for Th17 development, lymphocyte migration, and DC 
maturation [57-59]. Colonocytes benefit from a fat-rich diet, and mice treated with such a diet have reduced colitis [60], inflammatory bone disease, and bone erosion [61]. These mice have altered levels of gut Prevotella species as well as pro-IL-1 $\beta$ in neutrophils. Avoidance of certain microbe-metabolized nutrients may also help protect allograft health. In animal models, chronic dietary exposure of phosphatidylcholine, choline, or L-carnitine led to higher plasma TMAO levels in CKD subjects and was directly related to progressive renal fibrosis and cardiovascular disease [35, 38]. Although studies in renal transplant patients are limited, these observations suggest that dietary modifications may affect not only immune responses and microbiota composition, but also the availability of toxic metabolites.

Probiotics have been used in transplant recipients for the treatment of $C$.difficile. A retrospective study of lung and liver transplant recipients found Lactobacillus oral preparations to be safe and effective against C.difficile infection [62]. Lactobacillus $G G$ has shown efficacy, feasibility, and safety in preventing graft versus host disease in stem cell transplant recipients [63], although prospective studies are lacking. Oral Lactobacillus and Bifidobacterium were associated with improved intestinal barrier function, partial gut microbial restoration, and reduced liver injury after liver transplant in rats [64-65]. Whether probiotics affect outcomes other than C.difficile infection, and which probiotics are beneficial for renal transplant recipients,is unknown.

Restoration of microbiome diversity may be feasible through FMT. This was highly effective in patients with recurrent $C$.difficile colitis [66]; however, FMT in solid organ transplant has yet to be tested. FMT for $C$.difficile colitis was not associated with significant complications (such as bacteremia) in non-immunosuppressed [66] or immunosuppressed patients [67].

Precision microbiome restoration using only a single bacterial species has recently been reported by Buffie et al. In a murine model, the investigators treated mice with different antibiotic regimens and identified distinct changes in microbiota composition and C.difficile susceptibility among the groups. They used a cohort of immunosuppressed, antibiotictreated, stem-cell allotransplanted humans to identify native bacteria that displayed strong inhibition against $C$.difficile in both humans and mice. Clostridium scindens significantly enhanced resistance to $C$.difficile infection after adoptive transfer into mice, while transfer of other Clostridia species did not [68]. Recently, FMT in a kidney-heart transplant recipient with recurrent $C$.difficile was associated with restored microbiota diversity, improved clinical outcome, and loss of vancomycin-resistant enterococcus fecal dominance [69].

Certainly, avoidance of broad spectrum antibiotic use is essential to preserving microbial biodiversity and limiting development of resistance. Unfortunately, the immunosuppressed patient often requires empiric antimicrobial coverage to prevent overwhelming sepsis. Discovering ways to restore microbiota diversity or introduce essential microbial byproducts in this situation is thus highly desirable. 


\section{Conclusions}

Dysbiosis in the renal transplant patient may occur as a product of several contributing circumstances, including the effects of chronic kidney disease on systemic immunity and the gut microbiome; immunosuppression regimens; exposure to alloantigen and infection; and antimicrobial therapy. Microbiota affect changes in innate molecular defense mechanisms and shape $\mathrm{T}$ cell populations that can shift the immune climate toward pro- or antiinflammatory conditions. Microbiota thus represent an important point of regulation for immunomodulation in the transplant patient.

Shifts in the microbiota post-transplant have been recently identified; however, larger studies are needed to understand how microbiome alterations before and after transplantation can be used to predict clinical response to therapy, select those at risk for rejection or opportunistic infection, and design targeted therapies that promote health and graft tolerance.

\section{Abbreviations:}

C.diff

CKD

DAMPs

DC

eLSA

ESRD

FMT

IEC

ILC

iTreg

LefSe

LP

LTi

LPS

NASH

NIH

NOD1

SDD

Th
Clostridium difficile

chronic kidney disease

danger-associated molecular patterns

dendritic cells

extended local similarity analysis

end stage renal disease

fecal microbiota transplant

intestinal epithelial cells

innate lymphoid cells

induced Treg

linear discriminant analysis effect size

lamina propria

lymphoid tissue inducer cells

lipopolysaccharide

non-alcoholic steatohepatitis

National Institute of Health

nucleotide-binding oligomerization domain 1

selective digestive decontamination

T helper

Curr Opin Nephrol Hypertens. Author manuscript; available in PMC 2019 June 09. 

TLR
toll-like receptor
Treg
regulatory $\mathrm{T}$ cells
iTreg
induced regulatory $\mathrm{T}$ cell

\section{References}

Papers of particular interest, published within the annual period of review, have been highlighted as:

* of special interest

** of outstanding interest

1. Mulligan ME. Epidemiology of Clostridium difficile-induced intestinal disease. Rev. Infect. Dis 1984; 6:S222-S228. [PubMed: 6372032]

2. Stecher B, Chaffron S, Kappeli R, et al. Like will to like: abundances of closely related species can predict susceptibility to intestinal colonization by pathogenic and commensal bacteria. PLoS Pathog 2010; 6(1):e1000711. [PubMed: 20062525]

3. Wieland A, Frank DN, Harnke B. and Bambha K. Systematic review: microbial dysbiosis and nonalcoholic fatty liver disease. Alimentary pharmacology \& therapeutics 2015; 42(9):1051-1063. [PubMed: 26304302]

4. Wu HJ, Ivanov II, Darce J, et al. Gut-residing segmented filamentous bacteria drive autoimmune arthritis via T helper 17 cells. Immunity 2010; 32: 815-827. [PubMed: 20620945]

5. Bosco-Drayon V, Poidevin M, Boneca IG, et al. Peptidoglycan sensing by the receptor PGRP-LE in the Drosophila gut induces immune responses to infectious bacteria and tolerance to microbiota. Cell Host \& Microbe. 2012;12(2):153-65. [PubMed: 22901536]

6. Cebula A, Seweryn M, Rempala GA, et al. Thymus-derived regulatory T cells contribute to tolerance to commensal microbiota. Nature 2013; 497(7448):258-62. [PubMed: 23624374]

7. Gollwitzer ES, Saglani S, Trompette A, et al. Lung microbiota promotes tolerance to allergens in neonates via PD-L1. Nature Medicine 2014; 20(6):642-7.

8. Petersen C, Round JL. Defining dysbiosis and its influence on host immunity and disease. Cellular Microbiology 2015; 16: 1024-1033.

9. Manichanh C, Rigottier-Gois L, Bonnaud E, et al. Reduced diversity of faecal microbiota in Crohn's disease revealed by a metagenomic approach. Gut 2006; 55(2):205-21. [PubMed: 16188921]

10. Henao-Mejia J, Elinav E, Jin CC, et al. Inflammasome-mediated dysbiosis regulates progression of NAFLD and obesity. Nature 2012; 482: 179-U167. [PubMed: 22297845]

11. Weinstock GM. Genomic approaches to studying the human microbiota. Nature 2012; 489(7415): 250-256. [PubMed: 22972298]

12. McGarigal K, Cushman SA, Stafford S. Multivariate statistics for wildlife and ecology research. Springer Science \& Business Media 2013.

13. Xia LC, Steele JA, Cram JA, et al. Extended local similarity analysis (eLSA) of microbial community and other time series data with replicates. BMC systems biology. 2011; 5(2):1. [PubMed: 21194489]

14. Segata N, Izard J, Waldron L, et al. Metagenomic biomarker discovery and explanation. Genome Biol. 2011; 12(6):R60. [PubMed: 21702898]

15. National Institutes of Health. Human microbiome project 2015 [online] http:// commonfund.nih.gov/hmp.

16. MetaHIT. Metagenomics of the human intestinal tract 2012 [online] http://www.metahit.eu/.

17. Bouskra D, Brézillon C, Bérard M, et al. Lymphoid tissue genesis induced by commensals through NOD1 regulates intestinal homeostasis. Nature 2008; 456(7221):507-10. [PubMed: 18987631] 
18. Kanamori Y, Ishimaru K, Nanno M, et al. Identification of novel lymphoid tissues in murine intestinal mucosa where clusters of c-kit+ IL-7R+ Thy1+ lympho-hemopoietic progenitors develop. The Journal of Experimental Medicine. 1996; 184(4):1449-59. [PubMed: 8879216]

19. Clarke TB, Davis KM, Lysenko ES, et al. Recognition of peptidoglycan from the microbiota by Nod1 enhances systemic innate immunity. Nat Med. 2010; 16(2):228-231. [PubMed: 20081863]

20. Brandl K, Plitas G, Mihu CN, et al. Vancomycin-resistant enterococci exploit antibiotic-induced innate immune deficits. Nature 2008; 455(7214):804-807. [PubMed: 18724361]

21. Zeissig S, Blumberg RS. Commensal microbial regulation of natural killer T cells at the frontiers of the mucosal immune system. FEBS Lett. 2014; 588:4188-4194. [PubMed: 24983499]

22. Artis D, Spits H. The biology of innate lymphoid cells. Nature 2015;517(7534):293-301 [PubMed: 25592534]

23. Neish AS, Gewirtz AT, Zeng H, et al. Prokaryotic regulation of epithelial responses by inhibition of IxB-a ubiquitination. Science 2000; 289(5484):1560-3. [PubMed: 10968793]

24. Maynard CL, Elson CO, Hatton RD, Weaver CT. Reciprocal interactions of the intestinal microbiota and immune system. Nature. 2012; 489:231-241. [PubMed: 22972296]

25. Round JL, Mazmanian SK, Flavell RA. Inducible Foxp $3^{+}$regulatory T-cell development by a commensal bacterium of the intestinal microbiota. PNAS 2010; 10(27):12204-12209

26. Atarashi K, Tanoue T, Shima T, et al. Induction of colonic regulatory T cells by indigenous Clostridium species. Science 2011; 331:337-341. [PubMed: 21205640]

27. Ivanov II, Atarashi K, Manel N, et al. Induction of intestinal Th17 cells by segmented filamentous bacteria. Cell 2009; 139(3):485-498. [PubMed: 19836068]

28. Peck A, Mellins ED. Plasticity of T-cell phenotype and function: the T helper type 17 example. Immunology 2010; 129:147-153. [PubMed: 19922424]

29. Hudson KE, Lin E, Hendrickson JE, et al. Regulation of primary alloantibody response through antecedent exposure to a microbial T-cell epitope. Blood. 2010; 115(19):3989-96. [PubMed: 20086249]

30. Pantenburg B, Heinzel F, Das L, et al. T cells primed by Leishmania major infection cross-react with alloantigens and alter the course of allograft rejection. The Journal of Immunology 2002; 169(7):3686-93. [PubMed: 12244161]

31. Josefowicz SZ, Niec RE, Kim HY, et al. Extrathymically generated regulatory T cells control mucosal TH2 inflammation. Nature 2012;482(7385):395-9. [PubMed: 22318520]

32. Lupp C, Robertson ML, Wickham ME, et al. Host-mediated inflammation disrupts the intestinal microbiota and promotes the overgrowth of Enterobacteriaceae. Cell host \& microbe. 2007; 2(2) 119-29. [PubMed: 18005726]

33. Östman S, Rask C, Wold AE, et al. Impaired regulatory T cell function in germ-free mice. European journal of immunology. 20069 1;36(9):2336-46. [PubMed: 16897813]

34. Anders HJ, Anderson K, Stecher B. The intestinal microbiota, a leaky gut, and abnormal immunity in kidney disease. Kidney International 2013; 83: 1010-1016. [PubMed: 23325079]

35. Tang WW, Wang Z, Kennedy DJ, et al. Gut microbiota-dependent trimethylamine N-oxide (TMAO) pathway contributes to both development of renal insufficiency and mortality risk in chronic kidney disease. Circulation research. 2015; 116(3):448-55. [PubMed: 25599331] **This group has identified a novel mechanistic link between gut microbiota metabolism of dietary trimethylamine-containing nutrients and multiple clinical sequelae such as cardiovascular disease, thrombosis, and plateleat hyperactivity. This paper in particular shows a direct role of TMAO in the progression of renal fibrosis and dysfunction in animal models of CKD. Elevated plasma TMAO levels portend poor prognosis. This study shows how microbiota-derived metabolites can affect renal function.

36. Warrier M, Shih DM, Burrows AC, et al. The TMAO-generating enzyme flavin monooxygenase 3 is a central regulator of cholesterol balance. Cell reports. 2015; 10(3):326-38. [PubMed: 25600868]

37. Zhu W, Gregory JC, Org E, et al. Gut Microbial Metabolite TMAO Enhances Platelet Hyperreactivity and Thrombosis Risk. Cell. 2016; 165(1):111-24. [PubMed: 26972052]

38. Tang WH, Wang Z, Levison BS, et al. Intestinal microbial metabolism of phosphatidylcholine and cardiovascular risk. N Engl J Med. 2013; 368:1575-1584 [PubMed: 23614584] 
39. Wang F, Li Q, Wang C, et al. Dynamic alteration of the colonic microbiota in intestinal ischemiareperfusion injury. PLoS One 2012; 7:e42027. [PubMed: 22848694]

40. McIntyre CW, Harrison LE, Eldehni MT et al. Circulating endotoxemia: a novel factor in systemic inflammation and cardiovascular disease in chronic kidney disease. Clinical Journal of the American Society of Nephrology. 2011; 6(1):133-41. [PubMed: 20876680]

41. Anders HJ. Innate pathogen recognition in the kidney: toll-like receptors, NOD-like receptors, and RIG-like helicases. Kidney International 2007; 72: 1051-1056. [PubMed: 17653134]

42. Kiechl S, Lorenz E, Reindl M et al. Toll-like receptor 4 polymorphisms and atherogenesis. N Engl J Med 2002; 347: 185-192. [PubMed: 12124407]

43. Ranganathan N, Patel BG, Ranganathan $P$ et al. In vitro and in vivo assessment of intraintestinal bacteriotherapy in chronic kidney disease. ASAIO J 2006; 52: 70-79. [PubMed: 16436893]

44. Niwa T Role of indoxyl sulfate in the progression of chronic kidney disease and cardiovascular disease: experimental and clinical effects of oral sorbent AST-120. Ther Apher Dial 2011; 15:120124. [PubMed: 21426500]

45. Vaziri ND, Wong J, Pahl M, et al. Chronic kidney disease alters intestinal microbial flora. Kidney International. 2013; 83(2):308-15. [PubMed: 22992469]

46. Kato S, Chmielewski M, Honda H, et al. Aspects of immune dysfunction in end-stage renal disease. Clin J Am Soc Nephrol 2008; 3:1526-1533. [PubMed: 18701615]

47. Tchao NK, Turka LA. Lymphodepletion and homeostatic proliferation: implications for transplantation. Am J Transplant 2012; 12(5):1079-1090. [PubMed: 22420320]

48. Kieper WC, Troy A, Burghardt JT, et al. Recent immune status determines the source of antigens that drive homeostatic T cell expansion. J Immunol 2005 174:3158-3163. [PubMed: 15749843]

49. Oh PL, Martinez I, Sun Y, et al. 2012. Characterization of the ileal microbiota in rejecting and nonrejecting recipients of small bowel transplants. Am J Transplant 2012; 12:753-762. [PubMed: 22152019]

51. Willner DL, Hugenholtz P, Yerkovich ST, et al. Reestablishment of recipient-associated microbiota in the lung allograft is linked to reduced risk of bronchiolitis obliterans syndrome. Am J Respir Crit Care Med 2013; 187:640 [PubMed: 23328523]

52. Dickson RP, Erb-Downward JR, Freeman CM, et al. Changes in the lung microbiome following lung transplantation include the emergence of two distinct Pseudomonas species with distinct clinical associations. PLoS ONE 2014; 9(5):e97214 [PubMed: 24831685]

53. Fricke WF, Maddox C, Song Y, Bromberg JS. Human microbiota characterization in the course of renal transplantation. Am J Transplant 2014; 14:416-427. [PubMed: 24373208] **This is one of the first studies to characterize longitudinal changes in the microbiota in human kidney transplant patients. The results showed major changes in microbiota composition after transplantation.

54. Lee JR, Muthukumar T, Dadhania D, et al. Gut microbial community structure and complications after kidney transplantation: a pilot study. Transplantation 2014; 98:697-705. [PubMed: 25289916] **This prospective study also characterized the gut microbiota over three months of 26 kidney transplant recipients and found significant alterations in microbiota composition. They identified distinct microbiota alterations that were associated with post-transplant diarrhea, acute rejection, and Enterococcus UTI.

55. Lee JR, Muthukumar T, Dadhania D, et al. Gut microbiota and tacrolimus dosing in kidney transplantation. PLoS One 2015; 10:e0122399 10.1371/journal.pone.0122399. [PubMed: 25815766] *This study identified specific microbiota alterations that were predictive of future tacrolimus dosing at 1 month (fecal Faecalibacterium prausnitzii abundance). This observation showed that post-transplant dysbiosis may explain individual differences in pharmacokinetics and may identify earlier those patients who will need changes in their immunosuppression regimen.

56. Lemahieu W, Maes B, Verbeke K, et al. Cytochrome P450 3A4 and P-glycoprotein activity and assimilation of tacrolimus in transplant patients with persistent diarrhea. Am J Transplant. 2005; 5(6):1383-91. [PubMed: 15888045]

57. Cha HR, Chang SY, Chang JH, et al. Downregulation of Th17 cells in the small intestine by disruption of gut flora in the absence of retinoic acid. J Immunol 2010; 184: 6799-6806. [PubMed: 20488794] 
58. Chang SY, Cha HR, Chang JH, et al. Lack of retinoic acid leads to increased langerin- expressing dendritic cells in gut-associated lymphoid tissues. Gastroenterology 2010; 138:1468-1478, 1478.e1-1478.e6. [PubMed: 19914251]

59. Hall JA, Grainger JR, Spencer SP, Belkaid Y. The role of retinoic acid in tolerance and immunity. Immunity 2011; 35: 13-22. [PubMed: 21777796]

60. Devkota S, Wang Y, Musch MW, et al. Dietary-fat-induced taurocholic acid promotes pathobiont expansion and colitis in I110-/-mice. Nature 2012; 487(7405): 104-108. [PubMed: 22722865]

61. Lukens JR, Gurung P, Vogel P, et al. Dietary modulation of the microbiome affects autoinflammatory disease. Nature 2014; 516:246-249. 10.1038/nature13788 [PubMed: 25274309]

62. Deshpande A, Pasupuleti V, Mossad SB, et al. Use of Lactobacillus in Prevention of Recurrences of Clostridium difficile Infection in Solid Organ Transplant Recipients. Infectious Diseases in Clinical Practice, 2013; 21(5):292-298.

63. Gorshein E, Ambrosy S, Budney S, et al. Probiotic Enteric Regimen for Easing the Complications of Transplant. Blood 2014; 124(21): 5877-5877.

64. Ren Z, Liu H, Jiang J, et al. Protective effect of probiotics on intestinal barrier function in malnourished rats after liver transplantation. Hepatobiliary Pancreat Dis Int. 2011;10:489-496 [PubMed: 21947722]

65. Xie Y, Chen H, Zhu B, et al. Effect of intestinal microbiota alteration on hepatic damage in rats with acute rejection after liver transplantation. Microb Ecol. 2014; 68:871-880. [PubMed: 25004996]

66. van Nood E, Vrieze A, Nieuwdorp M, et al. Duodenal infusion of donor feces for recurrent Clostridium difficile. N Engl J Med 2013; 368(5):407-15 [PubMed: 23323867]

67. Kelly CR, Ihunnah C, Fischer M, et al. Fecal microbiota transplant for treatment of Clostridium difficile infection in immunocompromised patients. Am J Gastroenterol 2014; 109(7):1065-71 [PubMed: 24890442]

68. Buffie CG, Bucci V, Stein RR, et al. Precision microbiome reconstitution restores bile acid mediated resistance to Clostridium difficile. Nature 2015 517:205-208. 10.1038/nature13828. [PubMed: 25337874] **In this study, researchers used antibiotic-treated mice to characterize distinct microbial and metabolic environments and test their susceptibility to C.difficile infection. They observed that susceptibility to $C$.difficile after broad spectrum antibiotics was associated with loss of secondary bile acids, and this correlated with loss of specific microbiota members (Lachnospiraceae and Rnminococcaceae families). Restoration of secondary bile acids led to inhibition of spore germination and outgrowth in vitro. This suggests that future therapies for C.difficile may consider how microbiota regulates bile acids in the intestine.

69. Stripling J, Kumar R, Baddley JW, et al. Loss of vancomycin-resistant Enterococcus fecal dominance in an organ transplant patient with Clostridium difficile colitis after fecal microbiota transplant. Open Forum Infect Dis 2015; 2:ofv078 10.1093/ofid/ofv078. [PubMed: 26180828] 


\section{Key Points}

1. The microbiota interacts with the host in a bidirectional manner; microbes stimulate innate and adaptive mechanisms, and in turn require immunocompetence to suppress pathogenic bacteria and maintain gut homeostasis.

2. Gut dysbiosis may cause transfer of immune cells and signals to distant sites, rendering extra- intestinal tissues such as organ allografts susceptible to changes in the intestinal microbiota.

3. Dysbiosis may be associated with antigen cross reactivity due to molecular mimicry, potentially affecting the response to alloantigen. 


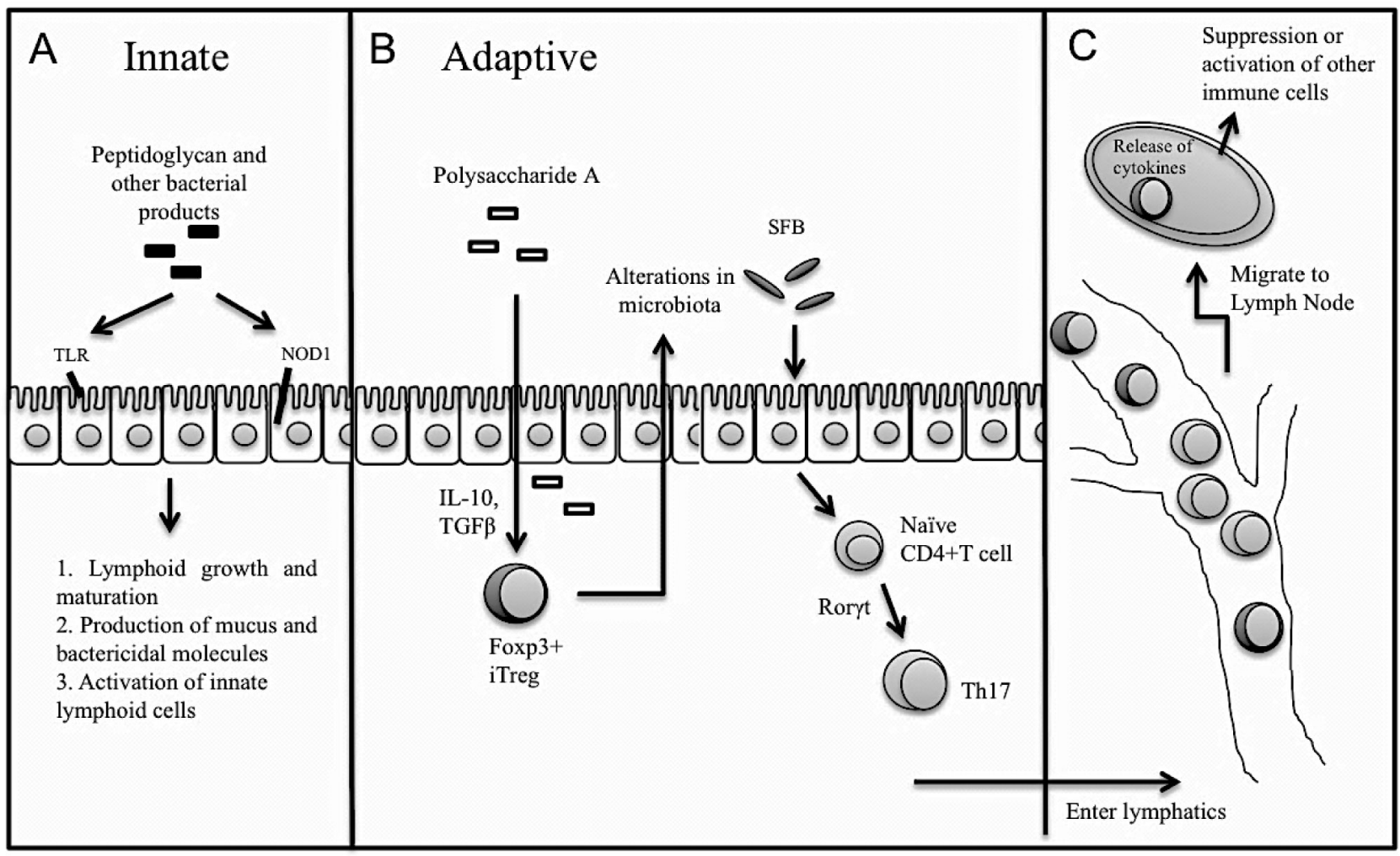

Figure 1.

Commensal signals such as peptidoglycan, segmental filamentous bacteria (SFB), and polysaccharide A either directly activate gut epithelial receptors (A) or indirectly stimulate differentiation of naïve CD4 T cells via cytokines such as TGF $\beta$ and Ror $\gamma$ t. Presence of differentiated CD4 T cells in turn influences microbiota composition (B). Effector T cells migrate via lymphatics to regional and systemic lymphoid tissue to interact with other immune cells (C). MHC, major histocompatibility complex; TLR, toll-like receptor. 
Table 1:

\section{Methods to study microbiota}

\begin{tabular}{|l|l|}
\hline 16S rRNA gene sequencing & $\begin{array}{l}\text { Distinct from eukaryotes, ribosomal RNA is used to identify bacterial and archaeal species based on } \\
\text { hypervariable regions. Provides only phylogenetic description of microbiome. }\end{array}$ \\
\hline Metagenomics & Genomic analysis of microorganisms by direct sequencing of DNA \\
\hline Shotgun RNA sequencing & Identifies transcriptional products of a microbiome. \\
\hline $\begin{array}{l}\text { Bioinformatics: } \\
\text { Principal coordinate analysis } \\
\text { Classical multidimensional scaling } \\
\text { Extended similarity analysis } \\
\text { Linear discriminant analysis effect size }\end{array}$ & Used to visualize similarities or dissimilarities in a complex data set. \\
\hline
\end{tabular}


Table 2:

Immunologic changes during chronic kidney disease (Anders 2013)

Increased production of pro-inflammatory cytokines

Increased complement activation

Activation of adhesion molecule expression in endothelial cells

Increased production of reactive oxygen species inducing oxidative stress and endothelial dysfunction

Decreased phagocytic function

Impaired antigen-presentation

Impaired ratio of CD4/CD8 T cells

Impaired B cell response

Shift of Th1/Th2 ratio toward Th2 cells 
Table 3:

Evidence for role of microbiota in chronic kidney disease

\begin{tabular}{|l|l|}
\hline $\begin{array}{l}\text { Tang et al 2015, Warrior et al. 2015; } \\
\text { Zhu et al. 2016, Tand et al. 2013 }\end{array}$ & $\begin{array}{l}\text { Gut microbial metabolite trimethylamine-N-oxide (TMAO) directly contributes to progressive renal } \\
\text { fibrosis and dysfunction in animal models; increases mortality risk in CKD; enhances platelet } \\
\text { hyperreactivity and thrombosis risk; regulates lipid metabolism and inflammation; TMAO may be } \\
\text { linked to specific dietary nutrients and gut microbes. }\end{array}$ \\
\hline Wang et al. 2012 & $\begin{array}{l}\text { Experimental uremia in rats increases bacterial translocation from gut, which is associated with higher } \\
\text { serum IL-6 and C-reactive protein levels. (Wang 2012) }\end{array}$ \\
\hline McIntyre et al. 2011 & $\begin{array}{l}\text { Progressive levels of circulating bacterial endotoxin and LPS with CKD are highest in patients on } \\
\text { dialysis. LPS levels are an independent predictor of mortality. }\end{array}$ \\
\hline Anders et al. 2007 & $\begin{array}{l}\text { Bacterial products activate pattern-recognition receptors on various immune cells inside and outside the } \\
\text { kidney. }\end{array}$ \\
\hline Kiechl et al. 2002 & Increased LPS/TLR-4 signaling may promote accelerated atherogenesis in CKD. \\
\hline Ranganathan et al. 2006 & Oral intake of nonpathogenic Sprosarina pasteurii improved renal function and survival of uremic rats. \\
\hline Niwa et al. 2011 & $\begin{array}{l}\text { Oral neutralization of indoxyl sulfate (a bacteria-derived uremic toxin) delays progression of CKD and } \\
\text { cardiovascular disease in uremic rats. }\end{array}$ \\
\hline
\end{tabular}

